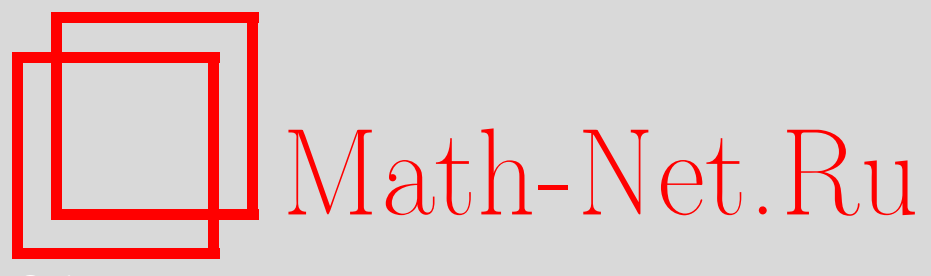

C. Е. Пастухова, С. В. Тихомирова, Эллиптическое уравнение с несимметрической матрицей. Усреднение "вариационных решений", Матем. заметки, 2007, том 81, выпуск 4, 631-635

DOI: https://doi.org/10.4213/mzm3707

Использование Общероссийского математического портала Math-Net.Ru подразумевает, что вы прочитали и согласны с пользовательским соглашением http://www . mathnet.ru/rus/agreement

Параметры загрузки:

IP: 34.239 .49 .27

26 апреля 2023 г., 15:09:33

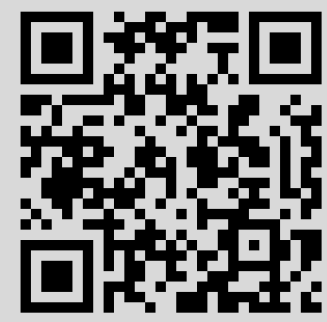




\section{Эллиптическое уравнение с несимметрической матрицей. Усреднение "вариационных решений"}

\section{С. Е. Пастухова, С. В. Тихомирова}

1. Рассмотрим стационарное уравнение диффузии во всем пространстве

$$
u^{\varepsilon} \in H^{1}\left(\mathbb{R}^{n}\right), \quad-\operatorname{div}\left(\nabla u^{\varepsilon}+B\left(\varepsilon^{-1} x\right) \nabla u^{\varepsilon}\right)+u^{\varepsilon}=f \in L^{2}\left(\mathbb{R}^{n}\right),
$$

где $B(y)$ - измеримая кососимметрическая периодическая матрица с ячейкой периодичности $\square=[-1 / 2,1 / 2)^{n}$. Под решением задачи (1) понимается функция $u^{\varepsilon}$, удовлетворяющая интегральному тождеству

$$
\int_{\mathbb{R}^{n}} A\left(\varepsilon^{-1} x\right) \nabla u^{\varepsilon} \nabla \varphi d x+\int_{\mathbb{R}^{n}} u^{\varepsilon} \varphi d x=\int_{\mathbb{R}^{n}} f \varphi d x \quad \forall \varphi \in C_{0}^{\infty}\left(\mathbb{R}^{n}\right),
$$

где $A(y)=\mathbf{1}+B(y), \mathbf{1}$ - единичная матрица. Данное определение имеет смысл, если $B \in$ $L_{\mathrm{per}}^{2}(\square)$. Это же условие обеспечивает существование решения, но не единственность. Доказательство разрешимости можно провести с помощью аппроксимации

$$
B_{N} \in L_{\mathrm{per}}^{\infty}(\square), \quad B_{N} \rightarrow B \quad \text { в } L_{\mathrm{per}}^{2}(\square), \quad B_{N} \text { кососимметрична. }
$$

C матрицей $B_{N}$ (вместо $B$ ) уравнение (1) разрешимо, последовательность его решений $u_{N}=u_{N}^{\varepsilon}$ равномерно ограничена в $H^{1}\left(\mathbb{R}^{n}\right)$, и можно считать, что $u_{N} \rightarrow u$ в $H^{1}\left(\mathbb{R}^{n}\right)$. Переход к пределу в интегральном тождестве показывает, что $u$ - решение исходного уравнения (1). Видим, что процедура аппроксимации (3) всякий раз приводит к решению задачи, которое принято называть аппроксимачионным. Условие $B \in L_{\mathrm{per}}^{2}(\square)$ не гарантирует единственности аппроксимационного решения. Кроме того, помимо аппроксимационных могут существовать другие решения, которые нельзя получить путем аппроксимаций. Пример уравнения типа (1) с подобного рода решением построен в [1].

В связи с неединственностью решения задачи (1) в работе [1] рассматривался особый способ однозначного выделения решения для уравнения типа (1), названный вариационным. Он заключается в следующем. Вводится операторная система

$$
\begin{aligned}
-\Delta u_{1}^{\varepsilon}+S u_{2}^{\varepsilon}+u_{1}^{\varepsilon} & =f_{1}, \\
-\Delta u_{2}^{\varepsilon}-S^{*} u_{1}^{\varepsilon}+u_{2}^{\varepsilon} & =f_{2},
\end{aligned} \quad u_{1}^{\varepsilon}, u_{2}^{\varepsilon} \in H^{1}\left(\mathbb{R}^{n}\right),
$$

где оператор $S$, действующий из $H^{1}\left(\mathbb{R}^{n}\right)$ в $H^{-1}\left(\mathbb{R}^{n}\right)$, определен сначала равенством

$$
(S u, \varphi)=\int_{\mathbb{R}^{n}} B \nabla u \nabla \varphi d x \quad \forall \varphi \in H^{1}\left(\mathbb{R}^{n}\right)
$$

для произвольной $u \in C_{0}^{\infty}\left(\mathbb{R}^{n}\right)$, а затем продолжен до замкнутого оператора. Несмотря на то, что форма (5) кососимметрична для $u, \varphi \in C_{0}^{\infty}\left(\mathbb{R}^{n}\right)$, оператор $S$ не обязательно кососамосопряженный, так как области определения $D=\operatorname{dom} S$ и $D^{*}=\operatorname{dom} S^{*}$, вообще говоря, не совпадают. Однако “матричный” оператор

$$
T=\left(\begin{array}{cc}
0 & S \\
-S^{*} & 0
\end{array}\right)
$$

Работа выполнена при поддержке Российского фонда фундаментальных исследований (грант № 05-01-00621). 
всегда кососамосопряжен на своей области определения $\operatorname{dom} T=\operatorname{dom} S^{*} \times \operatorname{dom} S=D^{*} \times D$ для любого замкнутого оператора $S$, в результате чего система (4) однозначно разрешима. В самом деле, ее можно записать в виде операторного уравнения

$$
(I+T)\left(\begin{array}{c}
u_{1}^{\varepsilon} \\
u_{2}^{\varepsilon}
\end{array}\right)=\left(\begin{array}{l}
f_{1} \\
f_{2}
\end{array}\right)
$$

где $I=-\Delta+1: H^{1}\left(\mathbb{R}^{n}\right)^{2} \rightarrow H^{-1}\left(\mathbb{R}^{n}\right)^{2}$, разрешимость которого нетрудно установить.

Если в (4) $f_{1}=f, f_{2}=0$, то сумма $u_{1}+u_{2}$ удовлетворяет тождеству (2) и, значит, является решением задачи (1). Это решение мы получили, минуя процедуру аппроксимации, и будем называть его вариационным.

Заметим, что решение системы (4) можно задать условиями: $u_{1}^{\varepsilon} \in H^{1}\left(\mathbb{R}^{n}\right), u_{2}^{\varepsilon} \in D$ и выполнено интегральное тождество

$$
\left\{\begin{array}{l}
\int_{\mathbb{R}^{n}}\left(\nabla u_{1}^{\varepsilon} \cdot \nabla \varphi_{1}+u_{1}^{\varepsilon} \varphi_{1}\right) d x+\int_{\mathbb{R}^{n}} B \nabla u_{2}^{\varepsilon} \nabla \varphi_{1} d x=\int_{\mathbb{R}^{n}} f_{1} \varphi_{1} d x, \\
\int_{\mathbb{R}^{n}}\left(\nabla u_{2}^{\varepsilon} \cdot \nabla \varphi_{2}+u_{2}^{\varepsilon} \varphi_{2}\right) d x+\int_{\mathbb{R}^{n}} B \nabla u_{1}^{\varepsilon} \nabla \varphi_{2} d x=\int_{\mathbb{R}^{n}} f_{2} \varphi_{2} d x,
\end{array} \quad \forall \varphi_{1}, \varphi_{2} \in C_{0}^{\infty}\left(\mathbb{R}^{n}\right) .\right.
$$

В самом деле, в силу тождества $(6)_{2} u_{1}^{\varepsilon} \in D^{*}$ и

$$
\int_{\mathbb{R}^{n}} B \nabla u_{1}^{\varepsilon} \nabla \varphi_{2} d x=-\left(S^{*} u_{1}^{\varepsilon}, \varphi_{2}\right)
$$

поэтому систему (6) можно записать в следующем виде:

$$
\left\{\begin{array}{l}
\int_{\mathbb{R}^{n}}\left(\nabla u_{1}^{\varepsilon} \cdot \nabla \varphi_{1}+u_{1}^{\varepsilon} \varphi_{1}\right) d x+\left(S u_{2}^{\varepsilon}, \varphi_{1}\right)=\int_{\mathbb{R}^{n}} f_{1} \varphi_{1} d x \\
\int_{\mathbb{R}^{n}}\left(\nabla u_{2}^{\varepsilon} \cdot \nabla \varphi_{2}+u_{2}^{\varepsilon} \varphi_{2}\right) d x+\left(-S^{*} u_{1}^{\varepsilon}, \varphi_{2}\right)=\int_{\mathbb{R}^{n}} f_{2} \varphi_{2} d x
\end{array}\right.
$$

причем в такой записи системы допускаются пробные функции $\varphi_{1} \in D, \varphi_{2} \in D^{*}$.

2. Теперь обратимся к задаче усреднения для уравнения (1). В условиях неоднозначной разрешимости будем для каждого $\varepsilon$ выделять его вариационное решение $u^{\varepsilon}$ указанным выше способом. Наша цель - найти предел последовательности $u^{\varepsilon}$ и указать уравнение, которому он удовлетворяет. Ясно, что для этого необходимо изучить процедуру усреднения для системы вида (4). Только этим вопросом мы далее и занимаемся, выделив его в отдельный объект исследования.

Разберем формальную процедуру усреднения для системы уравнений (4). Справедлива следующая

Лемма 1. Решение задачи (4) существует, единственно и удовлетворяет равномерной по в оценке

$$
\left\|u_{1}^{\varepsilon}\right\|_{H^{1}}^{2}+\left\|u_{2}^{\varepsilon}\right\|_{H^{1}}^{2} \leqslant\left\|f_{1}\right\|_{L^{2}}^{2}+\left\|f_{2}\right\|_{L^{2}}^{2} .
$$

Будем искать приближение к вариационному решению в виде

$$
\begin{aligned}
& \bar{u}_{1}^{\varepsilon}(x) \equiv \bar{u}_{1}(x, y)=u_{1}^{0}(x)+\varepsilon v_{1}(x, y), \quad y=\varepsilon^{-1} x . \\
& \bar{u}_{2}^{\varepsilon}(x) \equiv \bar{u}_{2}(x, y)=u_{2}^{0}(x)+\varepsilon v_{2}(x, y),
\end{aligned}
$$

Вычисления показывают, что

$$
\begin{aligned}
& I_{1} \equiv \nabla \bar{u}_{1}+B \nabla \bar{u}_{2}=\nabla u_{1}^{0}+\nabla_{y} v_{1}+B\left(\nabla u_{2}^{0}+\nabla_{y} v_{2}\right)+\varepsilon\left(\nabla_{x} v_{1}+B \nabla_{x} v_{2}\right), \\
& I_{2} \equiv \nabla \bar{u}_{2}+B \nabla \bar{u}_{1}=\nabla u_{2}^{0}+\nabla_{y} v_{2}+B\left(\nabla u_{1}^{0}+\nabla_{y} v_{1}\right)+\varepsilon\left(\nabla_{x} v_{2}+B \nabla_{x} v_{1}\right),
\end{aligned}
$$

и после подстановки (8) в (4) получаем следующие невязки в уравнениях:

$$
\left\{\begin{array}{c}
\varepsilon^{-1} \operatorname{div}_{y}\left[\nabla_{y} v_{1}+B\left(\nabla u_{2}^{0}+\nabla_{y} v_{2}\right)\right]+\operatorname{div}_{x}\left[\nabla u_{1}^{0}+\nabla_{y} v_{1}+B\left(\nabla u_{2}^{0}+\nabla_{y} v_{2}\right)\right] \\
\quad+\varepsilon \operatorname{div}\left(\nabla v_{1}+B \nabla_{x} v_{2}\right)+u_{1}^{0}+\varepsilon v_{1}-f_{1} \\
\varepsilon^{-1} \operatorname{div}_{y}\left[\nabla_{y} v_{2}+B\left(\nabla u_{1}^{0}+\nabla_{y} v_{1}\right)\right]+\operatorname{div}_{x}\left[\nabla u_{2}^{0}+\nabla_{y} v_{2}+B\left(\nabla u_{1}^{0}+\nabla_{y} v_{1}\right)\right] \\
\quad+\varepsilon \operatorname{div}\left(\nabla v_{2}+B \nabla_{x} v_{1}\right)+u_{2}^{0}+\varepsilon v_{2}-f_{2} .
\end{array}\right.
$$


Отсюда, приравнивая выражения при $\varepsilon^{-1}$ к нулю, получаем задачу на ячейке периодичности относительно $v_{1}(x, \cdot), v_{2}(x, \cdot)$

$$
\begin{gathered}
0=\operatorname{div}_{y}\left[\nabla_{y} v_{1}+B(y)\left(\nabla u_{2}^{0}+\nabla_{y} v_{2}\right)\right] \equiv \operatorname{div}_{y} S_{1}(x, y), \\
0=\operatorname{div}_{y}\left[\nabla_{y} v_{2}+B(y)\left(\nabla u_{1}^{0}+\nabla_{y} v_{1}\right)\right] \equiv \operatorname{div}_{y} S_{2}(x, y), \\
\left\langle v_{1}(x, \cdot)\right\rangle=0, \quad\left\langle v_{2}(x, \cdot)\right\rangle=0,
\end{gathered}
$$

где $\langle\cdot\rangle=\int_{\square} \cdot d y-$ среднее по ячейке, и условия нормировки добавлены для однозначного выделения решения. Заметим, что выражения $S_{1}(x, \cdot), S_{2}(x, \cdot)$ представляют собой соленоидальные векторы.

Очевидно, что вектор

$$
\left(\begin{array}{l}
v_{1} \\
v_{2}
\end{array}\right)
$$

линейно зависит от

$$
\left(\begin{array}{c}
\nabla u_{1}^{0} \\
\nabla u_{2}^{0}
\end{array}\right)
$$

т.е.

$$
v_{1}=N_{11}(y) \cdot \nabla u_{1}^{0}+N_{12}(y) \cdot \nabla u_{2}^{0}, \quad v_{2}=N_{21}(y) \cdot \nabla u_{1}^{0}+N_{22}(y) \cdot \nabla u_{2}^{0},
$$

где $N_{i j}-(2 \times 1)$-столбцы, составленные из компонент решений некоторых канонических задач вида (11), откуда следует представление

$S_{1}\left(y, \nabla u_{1}^{0}, \nabla u_{2}^{0}\right)=K_{11}(y) \nabla u_{1}^{0}+K_{12}(y) \nabla u_{2}^{0}, \quad S_{2}\left(y, \nabla u_{1}^{0}, \nabla u_{2}^{0}\right)=K_{21}(y) \nabla u_{1}^{0}+K_{22}(y) \nabla u_{2}^{0}$,

где $K_{i j}(y)$ - соленоидальные матрицы. По определению столбцами соленоидальной матрицы являются соленоидальные векторы.

Систему (11) можно переписать в виде уравнений

$$
0=\operatorname{div}_{y}\left[w_{1}+B(y)\left(\eta+w_{2}\right)\right], \quad 0=\operatorname{div}_{y}\left[w_{2}+B(y)\left(\xi+w_{1}\right)\right], \quad \zeta=\left(\begin{array}{l}
\xi \\
\eta
\end{array}\right) \in \mathbb{R}^{2 n}
$$

относительно вектора

$$
w \equiv w_{\zeta}=\left(\begin{array}{c}
w_{1} \\
w_{2}
\end{array}\right), \quad w_{i}=\nabla_{y} v_{i},
$$

где в качестве $\xi, \eta$ взяты $\nabla u_{1}^{0}, \nabla u_{2}^{0}$. Решение задачи (14) определяется так же, как для системы (4), через интегральные тождества типа (6), (7).

Лемма 2. 1) Задача (14) однозначно разрешима.

2) Пусть $(2 n \times 2 n)$-матрица $A^{0}$ определена равенством

$$
A^{0} \zeta=\left\langle\left(\begin{array}{cc}
\mathbf{1} & B \\
B & 1
\end{array}\right)\left(\zeta+w_{\zeta}\right)\right\rangle \quad \forall \zeta \in \mathbb{R}^{2 n},
$$

где $w_{\zeta}$ - решение задачи (14). Тогда $A^{0} \zeta \zeta \geqslant \zeta \cdot \zeta$.

Приравнивая к нулю выражения при нулевой степени в (10), получаем после взятия среднего по ячейке периодичности систему уравнений для $u_{1}^{0}, u_{2}^{0}$ :

$$
\begin{aligned}
& -\operatorname{div}_{x}\left[\nabla u_{1}^{0}+\left\langle S_{1}\left(x, \nabla u_{1}^{0}, \nabla u_{2}^{0}\right)\right\rangle\right]+u_{1}^{0}=f_{1}, \\
& -\operatorname{div}_{x}\left[\nabla u_{2}^{0}+\left\langle S_{2}\left(x, \nabla u_{1}^{0}, \nabla u_{2}^{0}\right)\right\rangle\right]+u_{2}^{0}=f_{2},
\end{aligned}
$$

которую символически можно записать так:

$$
\left\{\begin{array}{l}
-\operatorname{div}_{x} I_{1}^{0}+u_{1}^{0}=f_{1}, \\
-\operatorname{div}_{x} I_{2}^{0}+u_{2}^{0}=f_{2} .
\end{array}\right.
$$


Возможна запись системы (16) с помощью матрицы $A^{0}$ (см. (15))

$$
\begin{aligned}
u^{0} & =\left(\begin{array}{c}
u_{1}^{0} \\
u_{2}^{0}
\end{array}\right) \in H^{1}\left(\mathbb{R}^{n}\right)^{2}, \\
\int_{\mathbb{R}^{n}}\left(A^{0} \nabla u^{0} \nabla \varphi+u^{0} \cdot \varphi\right) d x & =\int_{\mathbb{R}^{n}} f \cdot \varphi d x \quad \forall \varphi=\left(\begin{array}{c}
\varphi_{1} \\
\varphi_{2}
\end{array}\right) \in C_{0}^{\infty}\left(\mathbb{R}^{n}\right)^{2},
\end{aligned}
$$

где

$$
f=\left(\begin{array}{l}
f_{1} \\
f_{2}
\end{array}\right), \quad \nabla u^{0}=\left(\begin{array}{c}
\nabla u_{1}^{0} \\
\nabla u_{2}^{0}
\end{array}\right) .
$$

Матрица $A^{0}$ имеет положительно определенную симметрическую часть, которая только и важна в этом уравнении. Поэтому справедлива

Лемма 3. Пусть $f \in L^{2}\left(\mathbb{R}^{n}\right)^{2}$, тогда существует и единственно решение задачи (17), причем $u^{0} \in H^{2}\left(\mathbb{R}^{n}\right)^{2}$ и выполнена оченка

$$
\left\|u^{0}\right\|_{H^{2}} \leqslant c\|f\|_{L^{2}}, \quad c=\left(n,\|B\|_{L^{2}(\square)}\right) .
$$

В принятых обозначениях (см. (9), (11), (17))

$$
\left\{\begin{array} { l } 
{ I _ { 1 } = \nabla u _ { 1 } ^ { 0 } + S _ { 1 } + \varepsilon ( \nabla _ { x } v _ { 1 } + B \nabla _ { x } v _ { 2 } ) , } \\
{ I _ { 2 } = \nabla u _ { 2 } ^ { 0 } + S _ { 2 } + \varepsilon ( \nabla _ { x } v _ { 2 } + B \nabla _ { x } v _ { 1 } ) , }
\end{array} \quad \left\{\begin{array}{l}
I_{1}^{0}=\nabla u_{1}^{0}+\left\langle S_{1}\right\rangle, \\
I_{2}^{0}=\nabla u_{2}^{0}+\left\langle S_{2}\right\rangle .
\end{array}\right.\right.
$$

Отсюда

$$
\left\{\begin{array}{l}
I_{1}-I_{1}^{0}=S_{1}-\left\langle S_{1}\right\rangle+\varepsilon\left(\nabla_{x} v_{1}+B \nabla_{x} v_{2}\right)=r_{1}, \\
I_{2}-I_{2}^{0}=S_{2}-\left\langle S_{2}\right\rangle+\varepsilon\left(\nabla_{x} v_{2}+B \nabla_{x} v_{1}\right)=r_{2},
\end{array}\right.
$$

где $S_{1}-\left\langle S_{1}\right\rangle, S_{2}-\left\langle S_{2}\right\rangle$ - соленоидальные по $y$ векторы (см. (11)), имеющие нулевое среднее.

Лемма 4 [2; гл. $1, \S 1]$. Если вектор $g \in L_{\text {per }}^{2}(\square), \operatorname{div} g=0,\langle g\rangle=0$, mо найдется кососимметрическая матрица $G \in H_{\mathrm{per}}^{1}(\square)$ такая, что $\operatorname{div}_{y} G=g$.

Получаем следующее представление (см. также (13)):

$$
S_{1}-\left\langle S_{1}\right\rangle=\Gamma_{11}(y) \nabla u_{1}^{0}+\Gamma_{12}(y) \nabla u_{2}^{0}, \quad S_{2}-\left\langle S_{2}\right\rangle=\Gamma_{21}(y) \nabla u_{1}^{0}+\Gamma_{22}(y) \nabla u_{2}^{0},
$$

где каждый столбец $g$ матрицы $\Gamma_{i j}$ имеет вид дивергенции от кососимметрической матрицы: $g=\operatorname{div} G, G_{i j}=-G_{j i}$. Рассмотрим подробно следующие за этим преобразования в правой части (20) на примере одного из слагаемых:

$$
\begin{gathered}
\Gamma_{11} \nabla u_{1}^{0}=g^{k} \frac{\partial u_{1}^{0}}{\partial x_{k}}, \\
g^{k}=\operatorname{div}_{y} G^{k}, \quad g^{k} \frac{\partial u_{1}^{0}}{\partial x_{k}} \equiv\left(\operatorname{div}_{y} G^{k}\right) \frac{\partial u_{1}^{0}}{\partial x_{k}}=\varepsilon \operatorname{div}\left(G^{k} \frac{\partial u_{1}^{0}}{\partial x_{k}}\right)-\varepsilon G^{k} \nabla\left(\frac{\partial u_{1}^{0}}{\partial x_{k}}\right),
\end{gathered}
$$

причем первое слагаемое в последней сумме есть соленоидальный вектор, что следует из кососимметричности матрицы $G^{k}$. Отсюда невязки $\operatorname{div} r_{1}, \operatorname{div} r_{2}$ (см. (19), (12)) содержат выражения вида

$$
\varepsilon \operatorname{div}\left(G^{j}(y) \nabla\left(\frac{\partial u_{i}^{0}}{\partial x_{j}}\right)\right), \quad \varepsilon \operatorname{div}\left(\nabla^{2} u_{i}^{0} N_{i j}(y)\right), \quad \varepsilon \operatorname{div}\left(B(y) \nabla^{2} u_{i}^{0} N_{i j}(y)\right) .
$$

Введем следующее основное предположение:

$$
B \in L_{\mathrm{per}}^{n}(\square) \quad \text { для } n \geqslant 3 \quad \text { и } \quad B \in L_{\mathrm{per}}^{2+\delta}(\square), \quad \text { где } \delta>0 \text {, для } n=2 .
$$

ЛЕмма 5. Если выполнено (22), то $B N_{i j} \in L_{\mathrm{per}}^{2}(\square)$. 
3. Нашей целью будет

Теорема 6. Пусть $u_{1}^{\varepsilon}, u_{2}^{\varepsilon}-$ решение задачи (4), $u_{1}^{0}, u_{2}^{0}-$ решение усредненной задачи (17). Тогда в предположении (22)

$$
\left\|u_{1}-u_{1}^{0}\right\|_{L^{2}}+\left\|u_{2}-u_{2}^{0}\right\|_{L^{2}} \leqslant c \varepsilon\left(\left\|f_{1}\right\|_{L^{2}}+\left\|f_{2}\right\|_{L^{2}}\right)
$$

где константа с зависит от размерности пространства и нормьь $\|B\|_{L_{\mathrm{per}}^{n}(\square)}$.

Дадим набросок доказательства этой теоремы.

Можно считать, что определенное в (8) первое приближение $\bar{u}_{1}^{\varepsilon}, \bar{u}_{2}^{\varepsilon} \in D^{*} \times D$ и поэтому является решением задачи вида (4) с подходящими правыми частями. Это соображение позволяет нам далее пользоваться энергетическим равенством и энергетической оценкой для уравнения (4).

По свойствам первого и нулевого приближений $\bar{u}_{1}^{\varepsilon}, \bar{u}_{2}^{\varepsilon}$ и $u_{1}^{0}, u_{2}^{0}$ имеем (см. (9), (17)-(19))

$$
\begin{aligned}
-\operatorname{div}\left(I_{1}-\nabla u_{1}^{\varepsilon}-B \nabla u_{2}^{\varepsilon}\right)+\bar{u}_{1}^{\varepsilon}-u_{1}^{\varepsilon} & =-\operatorname{div} I_{1}+\bar{u}_{1}^{\varepsilon}-f_{1}=-\operatorname{div} I_{1}+\bar{u}_{1}^{\varepsilon}+\operatorname{div} I_{1}^{0}-u_{1}^{0} \\
& =-\operatorname{div}\left(I_{1}-I_{1}^{0}\right)+\left(\bar{u}_{1}^{\varepsilon}-u_{1}^{0}\right)=-\operatorname{div} r_{1}+\varepsilon v_{1},
\end{aligned}
$$

аналогично

$$
-\operatorname{div}\left(I_{2}-\nabla u_{2}^{\varepsilon}-B \nabla u_{1}^{\varepsilon}\right)+\bar{u}_{2}^{\varepsilon}-u_{2}^{\varepsilon}=-\operatorname{div} r_{2}+\varepsilon v_{2} .
$$

Отсюда для $\varphi_{1}=u_{1}^{\varepsilon}-\bar{u}_{1}^{\varepsilon}, \varphi_{2}=u_{2}^{\varepsilon}-\bar{u}_{2}^{\varepsilon}$ энергетическое равенство имеет вид

$\int_{\mathbb{R}^{n}}\left(\varphi_{1}^{2}+\left|\nabla \varphi_{1}\right|^{2}\right) d x+\int_{\mathbb{R}^{n}}\left(\varphi_{2}^{2}+\left|\nabla \varphi_{2}\right|^{2}\right) d x=\int_{\mathbb{R}^{n}}\left(r_{1} \cdot \nabla \varphi_{1}-\varepsilon v_{1} \varphi_{1}\right) d x+\int_{\mathbb{R}^{n}}\left(r_{2} \cdot \nabla \varphi_{2}-\varepsilon v_{2} \varphi_{2}\right) d x$

и по свойствам $r_{i}, v_{i}$ (см. (12), (20), (21))

$$
\left\|\varphi_{1}\right\|_{H^{1}}^{2}+\left\|\varphi_{2}\right\|_{H^{1}}^{2} \leqslant c \varepsilon^{2} \int_{\mathbb{R}^{n}}\left|\theta\left(\varepsilon^{-1} x\right) \Phi(x)\right|^{2} d x
$$

где $\|\Phi\|_{L^{2}}^{2}=\left\|u_{1}^{0}\right\|_{H^{2}}^{2}+\left\|u_{2}^{0}\right\|_{H^{2}}^{2}$ и оценивается по лемме $3, \theta(y)$ - периодическая функция вида $N_{i j}(y), B N_{i j}(y), G^{j}(y) \in L_{\text {per }}^{2}(\square)$ (см. леммы 4,5). Прием, использованный в работах [3], [4], позволяет исключить из оценки (24) осциллирующие множители и получить в итоге неравенство (23).

\section{СПИСОК ЦИТИРОВАННОЙ ЛИТЕРАТУРЫ}

[1] В. В. Жиков, Функцион. анализ и его прилож., 38:3 (2004), 15-28. [2] В. В. Жиков, С. М. Козлов, О. А. Олейник, Усреднение дифференциальных операторов, Наука; Физматлит, М., 1993. [3] В. В. Жиков, Докл. РАН, 403:3 (2005), 305-508. [4] В. В. Жиков, С. В. Тихомирова, Современная матем. и ее прилож., 2005 (в печати).

\section{С. Е. Пастухова}

Московский институт радиотехники, электроники и автоматики

E-mail: leonowmw@cs.msu.su

\section{С. В. Тихомирова}

Владимирский государственный педагогический университет 